\title{
Why do we need another journal of pharmacogenomics research and personalized medicine?
}

The United States Food and Drug Administration defines personalized medicine as an approach which uses genetic or biomarker information to inform treatment-related decision-making. This may involve drug therapies being tailored to suit a patient's unique clinical characteristic profile in order to maximize safety and efficacy.

Personalized medicine, genetics, pharmacogenetics, and pharmacogenomics have all been used to refer to the study of genetic variation and how genetics affect therapeutic responses.

The past decade has witnessed a significant increase in publications in this field; however, there are many more studies to be published and there is much more work to be done.

\section{The field is expanding but is still underexplored}

Although genetic testing for drug therapy is a fast-growing research field, it is only in its infancy. The pharmacogenomic markers that have been validated so far account for only some of the variation seen in patients' clinical outcomes. Despite a number of guidelines for pharmacogenetics have been devised to date, they are not applied effectively in the clinical setting. Many scientists from around the globe are working together to address the challenges of implementing pharmacogenomics.

Pharmacogenomics Research and Personalized Medicine (PRPM) aims to fill this gap in the knowledge. PRPM will cover various aspects in the research of pharmacogenomics and personalized medicine, publishing articles that describe advances in basic and translational science, clinical findings and medical trials in relation to pharmacogenomics and personalized medicine.

\section{The need for more teamwork and network}

Pharmacogenomics holds great potential and has progressed significantly in the past few years. However, as stated above, the field is still in its infancy, and further research is needed to uncover the relationships of genes with therapeutic outcomes, as well as to verify preliminary findings, and to elucidate gene-drug relationships and devise corresponding prescribing guidance. International cooperation will play an essential role in achieving further developments in the field.

Within this setting, PRPM will provide an international and comprehensive platform for academics and practitioners worldwide to share and exchange ideas as well as further stimulate academic communication in the field in China and overseas.

\section{The need for a bridge between East and West}

PRPM is the official journal of Xiangya Hospital Central South University in Changsha in the south of China. The hospital is one of the oldest Western hospitals in China (founded in 1906 by the Yale-China Association of the United States). It ranks top in China in pharmacogenomics research and personalized medicine, and is home to the research team led by Prof. Honghao Zhou.

Given the unique origins of Xiangya Hospital, $P R P M$ will focus on East-West collaboration. This collaborative aspect will facilitate the development of a unique journal which will have international impact in the future. PRPM will take advantage of the collaboration between East and West to combine local expertise (in almost all sub-specialties) with international experience, which represents a unique feature of the journal. It will allow broad coverage but will also focus on special topics of interest through the publication of themed series.

\section{Adherence to high publication standards and transparency}

PRPM will adhere to the International Committee of Medical Journal Editors (ICMJE) recommendations for the Conduct, Reporting, Editing, and Publication of Scholarly Work in Medical Journal and the Committee on Publication Ethics (COPE)'s guidelines. Most importantly, PRPM will place great emphasis on publication quality and processing transparency, including but not limited to: 


\section{A transparent peer review process}

To commit to openness and accountability, and to increase the level of transparency throughout the peer review process, PRPM will implement a transparent peer review process as an option for all submissions. When a manuscript is submitted, authors will be asked (via the submission system) whether they agree to the publication of the "peer review file" (a record of reviewer reports and author replies) along with the accepted version should their article be accepted after the peer review process. The author's choice will not affect any subsequent editorial decisions.

The practice will see the inclusion of the peer review file in the footnote of the corresponding article. The reviewer's identity will still be kept confidential.

\section{Reporting guidelines for articles}

Each submission to PRPM will be required to follow the related EQUATOR research reporting guidelines and should include a reporting checklist. If an article is accepted, the corresponding reporting checklist will be published online with the article. For more details, please check the author instruction: https://prpm.amegroups.com/pages/view/guidelines-for-authors.

\section{Data sharing}

To keep pace with the data demands of the research field, PRPM will be committed to responsible data sharing in the setting of clinical trials and follow the Code of Conduct and Best Practice Guidelines for Journal Editors published by COPE.

Authors of primary research will be encouraged to state in the published paper whether and where data are available. Whenever applicable (e.g., when an original article includes any data that are not publicly available), authors will be required to fill in a data-sharing statement form, which should be submitted along with their manuscript. For articles which are accepted for publication, the Data Availability Statement (form) will be published online alongside the article.

PRPM will strive to follow the best practices in publishing.

The launch of PRPM is only part of the effort to advance pharmacogenomics research and personalized medicine. We believe $P R P M$ has the potential to develop into an internationally respected publication with unique local features. We look forward to you joining us on our journey!

\section{Acknowledgments}

Funding: None.

\section{Footnote}

Provenance and Peer Review: This article was commissioned by the editorial office, Pharmacogenomics Research and Personalized Medicine. The article did not undergo external peer review.

Conflicts of Interest: All authors have completed the ICMJE uniform disclosure form (available at http://dx.doi.org/10.21037/ prpm-2021-1). All authors serve as the editors-in-chief of Pharmacogenomics Research and Personalized Medicine. The authors have no other conflict of interest to declare.

Ethical Statement: The authors are accountable for all aspects of the work in ensuring that questions related to the accuracy or integrity of any part of the work are appropriately investigated and resolved. 
Open Access Statement: This is an Open Access article distributed in accordance with the Creative Commons AttributionNonCommercial-NoDerivs 4.0 International License (CC BY-NC-ND 4.0), which permits the non-commercial replication and distribution of the article with the strict proviso that no changes or edits are made and the original work is properly cited (including links to both the formal publication through the relevant DOI and the license). See: https://creativecommons.org/ licenses/by-nc-nd/4.0/.

Hong-Hao Zhou ${ }^{1,2}, \mathbf{M D}, \mathbf{P h D}$
${ }^{1}$ Department of Clinical Pharmacology, Xiangya Hospital, Central South University, Changsha, China;
${ }^{2}$ Institute of Clinical Pharmacology, Central South University, Changsha, China.
(Email: hbzhou2003@163.com)
Howard L. McLeod ${ }^{3}, \mathbf{P h D}$
${ }^{3}$ DeBartolo Family Personalized Medicine Institute, Moffitt Cancer Center, Tampa, FL, USA.
(Email: hmcleod@unc.edu)
Zhaoqian Liu', ${ }^{4}, \mathbf{M D}, \mathbf{P h D}$
(Email: Liuzhaoqian63@126.com)

Received: 30 March 2021; Accepted: 13 April 2021.

doi: $10.21037 / \mathrm{prpm}-2021-1$

View this article at: http://dx.doi.org/10.21037/prpm-2021-1

doi: $10.21037 /$ prpm-2021-1

Cite this article as: Zhou HH, McLeod HL, Liu Z. Why do we need another journal of pharmacogenomics research and personalized medicine? Pharmacogenomics Res Pers Med 2021. 